\title{
What should WHO be doing about patient safety and technology?
}

\author{
Oliver T Mytton, ${ }^{1,2}$ Felix E C Greaves, ${ }^{1,2}$ Emma A I Stanton, ${ }^{2}$ \\ Liam J Donaldson ${ }^{1,2}$
}

${ }^{1}$ WHO Patient Safety, Richmond House, London, UK

${ }^{2}$ Department of Health, Richmond House, London, UK

\section{Correspondence to} Dr Oliver Mytton, WHO Patient Safety, Richmond House, Richmond House, 79 Whitehall, London SW1A 2NL, UK; myttono@who.int

Accepted 8 April 2010

\section{INTRODUCTION}

Technology has promised a great deal as a tool for potentially reducing harm in healthcare, but has frequently failed to deliver. It can present risks to patients if used incorrectly. Technology can also mitigate against risk by reducing error through improved design of processes or equipment. ${ }^{-3}$ Technology is an important thread running through many areas of patient safety: reporting and learning, systems thinking, human factors, training and culture change. ${ }^{3}$

The opportunities for technology to improve patient safety are wide-ranging. Commonly described technological solutions include information technology, such as computerised physician order entry, ${ }^{45}$ as well as design solutions to make equipment safer to use, such as single-use injection needles. ${ }^{6}$ However, such examples fail to capture the full breadth of opportunities for technology to improve patient safety. ${ }^{1}$

At a macro level, regulation is required to ensure that new technology is introduced safely; ${ }^{7}$ however, regulation can also act as a barrier to innovation and lead to delays in implementation. New technological solutions can increase costs. At a micro level, workarounds, occurring on the introduction of technology intended to make care safer, can make care less safe. ${ }^{8}$ Healthcare professionals may resist the implementation of technological change. ${ }^{9}$

WHO Patient Safety is looking to develop a technology programme. As a first step to understand the relationship between technology and patient safety, WHO Patient Safety identified four key areas (box 1). Working with Imperial College London, they established four working groups of five to 10 experts for each of these areas. These groups sought to clarify the role of technology in improving patient safety, in both the developed and developing world.

Their reports are published as papers alongside this paper. ${ }^{2}>1011$ The group members producing these papers were invited to a 1-day conference, held at Imperial College in May 2009 (the WHO expert meeting), to present their work. In addition, at this expert meeting, the groups were invited to present two suggested projects to WHO Patient Safety, within their area. These proposals are presented in this paper. This paper draws on this work (the WHO expert meeting, the papers and the project proposals) and considers the wider themes emerging from this work. The paper, together with the group papers, provides the foundation for the next stage of the technology programme.

\section{EVALUATION OF PROJECT PROPOSALS}

Each of the four working groups proposed two projects to WHO Patient Safety. The working groups were advised that their projects would be considered alongside the priorities of WHO Patient Safety. Projects might then be undertaken by WHO Patient Safety as part of the next phase of its technology programme. The eight project proposals are shown in table 1.

Delegates from the four working groups attended the WHO expert meeting, where they presented their proposals. This expert meeting consisted of a series of break-out discussions in small groups to identify the strengths and weakness of each project. Each delegate at the expert meeting ranked each project on a five-point scale, against five criteria:

- burden of the problem;

- global nature of the solution and problem;

- potential for the solution to solve the problem;

- value for money;

- ability to use existing technology to solve the problem.

Scores on the five criteria were summed, with each criterion being given equal weight. The summed scores were averaged. Overall, the two simulation projects scored the highest. Both of the simulation projects had immediate face validity and addressed problems of global importance. The handover project was the most highly recommended project; delegates at the WHO expert meeting felt that poor communication at handovers was a global concern. It was proposed that a structured education tool be developed to promote uptake. It was suggested that such a tool could have worldwide impact, as the problem is universal, and would use low-fidelity simulation, making it easy to adapt or transfer at lower cost.

\section{CHALLENGES FOR TECHNOLOGY TO IMPROVE PATIENT SAFETY}

Several themes emerged from the group papers, ${ }^{27} 1011$ the $\mathrm{WHO}$ expert meeting and the project proposals. Those of particular interest are discussed.

\section{Theme 1: information management to understand errors}

We live in an information age. The total volume of digital data is predicted to increase more than five times by $2012 .{ }^{12}$ The challenge for patient safety is twofold. First, to manage the rapidly increasing volume of data so that healthcare professionals have access to the right information at the right time to provide safe and timely care. Second, to use, 
Box 1 WHO Patient Safety Technology Programme: priority-setting exercise

WHO Patient Safety and its stakeholders undertook a scoping exercise to identify and clarify the role of technology in improving patient safety in both the developed and developing world. Four working groups of international experts were created. Group members had relevant expertise in either technology or patient safety and reflected the different needs of developed and developing countries.

The four groups were:

- introducing new technology safely

- making existing technology safer in healthcare

- information technology for patient safety

- training and simulation for patient safety

and often share, electronically captured information to facilitate learning and improvements in patient safety. These themes are strongly echoed in the information technology paper, focussing on information transaction. ${ }^{2}$ Such changes take place against a backdrop of concerns about data protection ${ }^{13} 14$ and a growing enthusiasm for some patients to own their medical records.

Much hope is placed in Electronic Medical Records (EMR). ${ }^{3}$ Brigham and Women's Hospital in Boston, USA reduced serious medication errors by $55 \%$ following the introduction of computerised physician order entry systems, and had estimated savings of US $\$ 5-10$ million each year. ${ }^{15}$ The US Department of Veterans Affairs has doubled vaccination rates, and achieved substantial improvements in diabetes and acute coronary care, by changing to electronic records. ${ }^{16}$ However, the extent to which EMR have delivered outside a handful of settings remains unclear. Uptake in both ambulatory care and hospital care in the USA has been poor. ${ }^{17}$ Although one-quarter of office-based doctors in the USA reported using EMR systems, less than 10\% of these physicians actually have a 'complete EMR system. ${ }^{.17}$ Improving interconnectivity and the quality of data is vital to optimise their use. ${ }^{2}$ Improving data quality by identifying a practical set of quality measures for clinical coding (project three) reflects this theme.

The second challenge identified is the use of IT to facilitate efficient reporting and learning. The healthcare community has been set the challenge of passing the 'orange wire test': the airline industry has the ability to identify and rectify a systematic fault in a single orange wire across all aeroplanes within a period of days. ${ }^{18}$ Passing the orange wire test depends on an ability to identify problems rapidly, through data capture, pooling and analysis, as well as having systems for disseminating that information to those who can rectify the problem. Other high-hazard industries also carry out real-time analysis and reporting. Dow Corning, an international chemical company, has been widely praised for its safety record. It has a global incident management system that effectively uses IT to automatically collate, process and disseminate safety data in real time. $^{19}$

Approximately 30 years ago, experts in pharmacovigilance realised that a new approach was needed to identify drugs causing adverse events. ${ }^{20}$ Systems such as 'i3 Aperio' in the USA can be used to routinely scan electronic records for adverse events associated with the use of new medications or vaccines. The frequency of adverse events can be compared with similar patients in the same database who have not used the new medication or vaccine. Reports are run on a weekly basis to identify medication risks earlier than otherwise might be the case. Given the complexity of healthcare and the torrent of data, automated real-time analysis using information technology offers the best opportunity for healthcare systems to pass the orange wire test. Rapid dissemination of information is important, reflected in project 4 , to share best practice.

The importance of communicating information, particularly at transitions in care, is reflected in project 1 , simulation to improve communication at handovers. There are particular challenges for developing countries. The wide digital divide among countries shows little sign of narrowing. ${ }^{21}$ There is interest in using mobile phones to deliver information and coordinate action in the developing world; ${ }^{22-24}$ however, the quality and speed of information transfer by mobile phones are limited. A more pragmatic approach might be to encourage and develop broader global access to the internet.

\section{Theme 2: translating information on errors into safer technology}

In 2001, teenager Wayne Jowett died from vincristine poisoning. Wayne was in remission from leukaemia. Vincristine was injected intrathecally by mistake by two young doctors, rather than intravenously. One approach, employed in the UK, to prevent this happening is to improve awareness and training to change practice. To date, there have been no reported incidents of vincristine being administered in the UK by the wrong route since Wayne Jowett's tragic death. The National Patient Safety Agency (NPSA, UK) also reports there have been no near misses. Further incidents have continued to occur worldwide. ${ }^{25}{ }^{26} \mathrm{~A}$ second 'harder solution' of separate lock and key devices for intravenous and intrathecal injections creates a physical barrier to prevent inadvertent administration of intravenous drugs into the spine. Although various devices have been proposed, none has yet reached the market. This reflects a wider problem that effective solutions are not readily developed and introduced. The NPSA recently issued an alert mandating all NHS trusts to use spinal and epidural injection devices that cannot be connected to intravenous systems by $2013 .{ }^{27}$ Such regulation creates a market for safety devices. Greater use of financial incentives, such as national level purchasing contracts, could stimulate the development of safer technologies. More innovative approaches to collecting information on error, such as video recording of cardiac arrests, combined with an understanding of ergonomics and human factors, can produce radically different and safer designs. $^{28}$

There was concern expressed at the expert meeting about the lack of 'appropriate technology' designed specifically for developing countries. Appropriate technology is designed with special consideration to the environmental, ethical, cultural, social, political and economical aspects of the community for which it is intended. ${ }^{29}$ At the WHO expert group meeting, market failure was suggested as a possible root cause. An alternative approach to translating information on errors into safer technology is to give responsibility for innovation to frontline healthcare staff. This so-called 'lean' approach, pioneered by Toyota, has potential to deliver tailored economical solutions with greater speed. ${ }^{30}$ Lean thinking has been adopted into healthcare with limited success. ${ }^{31}$

\section{Theme 3: dissemination of technology}

Inconsistent uptake of medical advances is widely acknowledged. One study estimated that it took 17 years to apply only 
Table 1 Proposed projects assessed by the delegates

\begin{tabular}{|c|c|c|}
\hline Group & Project name & Brief description \\
\hline Introducing new technology safely & 1. Design & Greater use of appropriate technology in low-resource settings to promote patient safety \\
\hline Introducing new technology safely & 2. Governance & $\begin{array}{l}\text { Dual standards for technology in high- and low-income countries to facilitate the use of appropriate } \\
\text { technology in developing countries }\end{array}$ \\
\hline Making existing technology safer & 3. Implementation of technology & $\begin{array}{l}\text { Establish ways that technology could promote the safer use of medical devices in rural hospitals in } \\
\text { developing countries }\end{array}$ \\
\hline Making existing technology safer & 4. Integrated technology & $\begin{array}{l}\text { Assess the cost-effectiveness of using integrated technology to promote safer patient care in } \\
\text { developing countries }\end{array}$ \\
\hline Information technology & 5. Data quality & $\begin{array}{l}\text { Improve data quality in electronic records by identifying a practical set of quality measures for } \\
\text { clinical coding }\end{array}$ \\
\hline Information technology & 6. Forum for sharing best practice & Create a virtual international forum to promote learning and sharing of best practice \\
\hline Simulation & 7. Handover simulation & Develop a standard handover protocol, using simulation as the tool to teach its use \\
\hline Simulation & 8. Birthing simulator & Develop a simulator and program to promote safe childcare delivery \\
\hline
\end{tabular}

$10 \%$ of knowledge that could be beneficial to patients. ${ }^{32}$ This theme is echoed in several of the papers. 127

While other industries have managed to disseminate technological advances quickly and widely, healthcare has struggled to achieve the same success. This is partly due to the complexity of healthcare, with its fragmented structure, and partly due to an organisational culture that is resistant to change. It seems likely that different solutions are required in different settings. In developing countries, poor infrastructure hinders the delivery of care in the community. Over the past 40 years, US\$3.4 trillion has been spent to address critical diseases in developing countries; $^{33}$ however, the impact of this money is limited by the "last mile' distribution capabilities, leading to inequitable distribution of resources. ${ }^{33}$ Technology has a key role to play in communication, not simply by using new media for communication, but also by targeting messages to the end user. Organisations such as Amazon and Google tailor content by observing users' webbrowsing preferences. A similar approach could be incorporated into a virtual international forum to promote learning and sharing of best practice (project 4).

\section{Theme 4: regulation}

Any attempt to use technology to improve patient safety will need to tackle the challenge of regulation. Regulation supports the safe introduction of technology by ensuring the quality and safety of new devices; ${ }^{7}$ however, it can also delay or prevent safer innovative technology from wider use. ${ }^{7}$ Concern was expressed at the WHO expert meeting about how regulation affects developing countries. Regulatory systems are often absent or weak in developing countries allowing misguided use of technology. ${ }^{7}$ The lack of recognised regulatory bodies for these countries means that medications and devices intended solely for developing countries, particularly when deployed by international organisations, are mandated to pass a regulatory assessment. This has to occur in a developed country. There is little incentive for manufacturers to develop devices solely for developing countries, because the costs associated with regulatory assessment and the necessary over engineering are prohibitive. This theme was reflected in project 8, which proposed different regulatory standards for developing and developed countries.

A dual standard could be unethical, as it might promote differential rights to safe care for people living in different countries. However, the absence of dual standards might be acting to prohibit the introduction of appropriate technologies into developing countries. ${ }^{34}$ A different regulatory structure, or at least a different regulatory decision for different environments, may be seen as ethical, as it could facilitate the intro- duction of appropriate technology leading to better and safer healthcare in those settings. Different regulatory decisions would seem reasonable, given a different background incidence of disease, tolerance of error and funding possibilities in the developing world. A similar debate has taken place in research. ${ }^{35}$ The original standard was that any new innovation had to be tested against the best standard worldwide. This inhibited research to show the benefit of new technology or medicines that conferred a benefit in a particular setting where there might be other constraints preventing the routine use of the world 'gold standard'. The modern test is the local gold standard. This allows research into appropriate and affordable solutions for the setting.

One theme emerging from the WHO expert meeting was the need to understand human factors and ergonomics to produce smarter designs. Devices are often regulated in isolation and tested under ideal conditions, rather than realistic work conditions. Healthcare practitioners, particularly in developed countries, are also heavily regulated. However, no body 'regulates' the interaction between technology and healthcare practitioners. Most medical errors occur not because of individual failure or 'device failure' (a device not doing the task it was designed for), but because of how the design was used in the real world (the interaction between healthcare practitioner and technology). ${ }^{11}$ The need for a greater consideration of device-user interaction is reflected in projects 5, 6 and 7. Simulated ward environments could be used more routinely to develop devices to promote patient safety and feed directly into regulatory decisions.

\section{Theme 5: using technology to improve safety in developing countries}

There have been significant technological advances in healthcare in the most affluent nations, but concern was expressed at the expert meeting that their spread to developing countries has been poor. Funding is a crucial issue. Donor money can establish a market, as occurred with fixed-dose combination therapy for tuberculosis ${ }^{36}$; however, in the present economic climate, the sustainability of such projects remains uncertain. ${ }^{37}$

There is a paucity of appropriate technology for developing countries, reflected by the proposal in project 7 . Donations of developed world technology are often unsuitable for local needs and left unused. ${ }^{78}$ Technology designed for the developed world is often too expensive and is often poorly designed for the environment where it will be used. These technologies might be overly complicated, difficult to maintain or incompatible with other devices. 711

Education is important for sustainable development in developing countries, ${ }^{39}$ as well as being vital for improving 
healthcare. The two simulation projects reflect this priority. Simulation is a hands-on way of learning, which could be well suited to the developing world.

\section{CONCLUSION: WHO PATIENT SAFETY TECHNOLOGY PROGRAMME, A LONG-TERM VISION}

Technology is, and will continue to be, an important theme for patient safety. For WHO Patient Safety to take a lead internationally in improving patient safety, it needs to continue to incorporate technology within its current thinking and future work. The technology programme will support WHO Patient Safety in this role.

The WHO expert meeting, and the four papers presented here, ${ }^{2} 71011$ highlighted the breadth and complexity of the relationship between technology and patient safety. It will be challenging for WHO Patient Safety alone to develop a technology programme that has sufficient breadth and depth of skill to support the needs of its other programmes. A more flexible approach is required that can tap into a wide global and diverse skill matrix in academia, industry and policy.

The technology programme, like WHO Patient Safety, needs to balance implementation, delivering projects with measurable outcomes, with thought leadership and standard setting. The latter role might be more influential, pushing the boundaries and challenging present thinking. Such a balance can be achieved by choosing original and innovative projects that demonstrate new approaches, yet still support other areas of WHO Patient Safety's work.

In the short term, both of the simulation projects, concerning safe childbirth and promoting safe handovers, are of great interest. These projects align with other areas of work by WHO Patient Safety. The technology programme should support WHO Patient Safety in its aim to promote and make patient care safer throughout the developed and developing world. This principle should remain at the heart of future developments for the technology programme.

Fundings The project was funded by WHO Patient Safety.

Competing interests LJD is the Chair of WHO Patient Safety and the Chief Medical Officer for England. From July 2010, he will become the Chair of National Patient Safety Agency in the UK. OTM, FECG and EAIS have all been clinical advisors to LJD, working for either WHO Patient Safety or the Department of Health.

Contributors OTM drafted the manuscript in consultation with EAIS, LJD and FECG. All reviewers critiqued the manuscript. OTM is guarantor.

Provenance and peer review Not commissioned; externally peer reviewed.

\section{REFERENCES}

1. Mytton OT, Greaves FEC, Stanton EAl, et al. What should WHO be doing about patient safety and technology? Qual Saf Health Care 2010;

19(Suppl 2):44-47.

2. Huckvale C, Car J, Akiyama M, et al. Information technology for patient safety. Qual Saf Health Care 2010:19(Suppl 2):25-33.

3. Bates D, Gawande A. Improving safety with information technology. N Engl J Med 2003;348:2526-34.

4. Bates DW, Leape LL, Cullen D, et al. Effect of computerized physician order entry and a team intervention on prevention of serious medication errors. JAMA 1998;280:1311-16.

5. Bates DW, Teich JM, Lee J, et al. The impact of computerised physician order entry on medication error prevention. J Am Med Inform Assoc 1999;6:313-21.

6. Steinglass R, Boyd D, Grabowsky M, et al. Safety, effectiveness and ease of use of a non-reusable syringe in a developing country immunization programme. Bull World Health Organ 1995;73:57-63.
7. The Economist. A doctor in your pocket. London, UK: The Economist, 2009.

8. Patterson ES, Rogers ML, Chapman RJ, et al. Compliance with intended use of Bar Code Medication Administration in acute and long-term care: an observational study. Hum Factors 2006;48:15-22.

9. Mytton OT, Velazquez A, Banken R, et al. Introducing new technology safely. Qual Saf Health Care 2010;19(Suppl 2):9-14.

10. Aggarwal R, Mytton OT, Derbrew M, et al. Training and simulation for patient safety. Qual Saf Health Care 2010;19(Suppl 2):34-43.

11. Newton R, Mytton OT, Aggarwal R, et al. Making existing technology safer in healthcare. Oual Saf Health Care 2010;19(Suppl 2):15-24.

12. New York Times. For today's graduates one word: statistics. http://www.nytimes com/2009/08/06/technology/06stats.html (accessed 20 Aug 2009).

13. Harrison D. The Government's record year of data loss. London: The Daily Telegraph 2008. http://www.telegraph.co.uk/news/newstopics/politics/1574687/Governmentsrecord-year-of-data-loss.html (accessed 28 Nov 2009).

14. McGee MK. Laptop stolen with personal data on 300,000 health insurance clients Information Week, New York, 2008.http://www.informationweek.com/news/ security/showArticle.jhtml?articlelD=206100526 (accessed 28 November 2009)

15. A State Policy Approach: Promoting Health Information Technology in California (2007) Legislative Analyst's Office. http://www.lao.ca.gov/2007/health info tech/ health info tech 021307.aspx (accessed 14 Dec 2009)

16. Jha $\overline{\mathbf{A K}}$, Perlin $\overline{\mathrm{JB}}$, Kizer $\mathrm{KW}$, et al. Effect of the transformation of the Veterans affairs health care system on the quality of care. N Engl J Med 2003;348:2218-27.

17. Jha AK, DesRoches CM, Campbell EG, et al. Use of electronic health records in US hospitals. N Engl J Med 2009;360:1628-38.

18. Donaldson $\mathbf{L}$. When will health care pass the orange-wire test? Lancet 2004;364:1567-8.

19. Morrow ML. Dow Corning Corporation's Auburn Site Receives State's Highest Safety and Health Award. Michigan, USA: Department of Energy, Labor \& Economic Growth, 2007. http://www.michigan.gov/dleg/0,1607,7-154-10573_11472196834-00 html.

20. Jick H, Walker AM, Spriet-Pourra C. Postmarketing follow-up. JAMA 1979:242:2310-14.

21. International Telecommunication Union. Measuring the information society. Geneva: ITU, 2009.

22. Lester R, Karanja S. Mobile phones: exceptional tools for HIV/AIDS, health, and crisis management. Lancet Infect Dis 2008;8:738-9.

23. Idowu B, Adagunodo R, Adedoyin R. Information technology infusion model for health sector in a developing country: Nigeria as a case. Technol Health Care 2006;14:69-77.

24. Chang LW, Kagaayi J, Nakigozi G, et al. Responding to the human resource crisis peer health workers, mobile phones, and HIV care in Rakai, Uganda. AIDS Patient Care STDS 2008;22:173-4.

25. D'Addario A, Galuppo J, Navari C, et al. Accidental intrathecal administration of Vincristine. Am J Forensic Med Pathol Published online first: 20 November 2009.

26. Hennipman B, de Vries E, Bokkerink JPM, et al. Intrathecal Vincristine: 3 fatal cases and a review of the literature. J Pediatr Hematol Oncol 2009;31:816-9.

27. NPSA. Safer spinal (intrathecal), epidural and regional devices. London: NPSA, 2009 http://www.nrls.npsa.nhs.uk/resources/type/alerts/?entryid45=65259 (accessed 28 Nov 2009)

28. West J, Halls $\mathrm{S}$, Coleman $\mathrm{R}$, et al. Resus-station: breathing life back into resuscitation. London, UK: Helen Hamlyn Centre, 2008. http://www.hhc.rca.ac.uk/ cms/files/JWestResusStation.pdf (accessed Apr 2010).

29. Wikipedia. Appropriate technology. http://en.wikipedia.org/wiki/ Appropriate technology (accessed 10 Feb 2010).

30. Womack JP, Jones DT, Roos D. The machine that changed the world. New York: Simon and Schuster Ltd, 2007.

31. Eaton M. Lean for practitioners: an introduction to lean for healthcare organisations UK: Ecademy Press, 2008

32. Balas EA, Boren SA. Managing clinical knowledge for health care improvement Yearbook of Medical Informatics 2000:65-70.

33. Village Reach. http://villagereach.org/challenges-at-the-last-mile/last-milechallenges/ (accessed 17 Dec 2009).

34. Milstien J, Cash RA, Wecker J, et al. Development of priority vaccines for diseaseendemic countries: risk and benefit. Health Aff (Millwood) 2005;24:718-28.

35. Wikler D, Pang T. Developing-world research ethics. Lancet 2009;355:70.

36. WHO. Global pulse oximetry — background document. Geneva, Switzerland: WHO, 2008. http://www.who.int/patientsafety/events/08/ 1st pulse oximetry meeting background doc.pdf (accessed Apr 2010)

37. Brock DW $\bar{W}$, Wikler $\bar{D}$. Ethical challenges in long-term funding for HIV/AIDS. Health Aff (Millwood);28:1666-76

38. Peña-Mohr J. Distributing and transferring medical technology. A view from Latin America and the Caribbean. Int J Technol Assess Health Care 1987;3:281-91.

39. United Nations Education, Scientific and Cultural Organization. Os/o Declaration: eighth meeting of the high-level group on education for all. Unesco, 2008. 